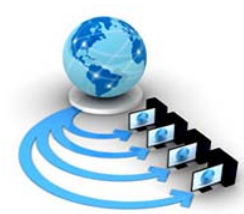

Volume 10, No. 6, November-December 2019

\title{
OPTIMIZATION AND RESEARCH ON HYDRAULIC CONTROL SYSTEM OF MACHINING EQUIPMENT WORKTABLE
}

\author{
Shengwei SUN \\ Binzhou University \\ Binzhou, China
}

\begin{abstract}
In order to solve the problem of precise positioning at micron level, a hydraulic system using servo motor and displacement amplifier hydraulic cylinder instead of servo valve is proposed. The simulation results show that the open-loop response speed of the system can be improved by increasing the elastic modulus of the oil and reducing the viscosity of the oil, while the pressure and volume of the accumulator have no obvious influence on the open-loop performance of the system. The micro displacement control hydraulic system is applied to the integrated test-bed, and PD controller is selected as the position controller according to the identification results. The validity and feasibility of the micro displacement control hydraulic system are verified.
\end{abstract}

Keywords: hydraulic control system, simulation, elastic modulus, micron level

\section{INTRODUCTION}

The hydraulic control system is an indispensable part of many machine tools, which has a key impact on the machining accuracy of the machine, such as the power slide hydraulic system of the combined machine tool, the hydraulic system of the press, the hydraulic system of the multi cylinder milling machine, etc [1]. Micro stroke control ability is one of the key indexes to measure the machining ability and overall technical level of machine tools. Because the traditional hydraulic system is usually based on oil pressure or flow control, the machining process is easy to be affected by the nonlinear factors of hydraulic valves and hydraulic pumps, so it is necessary to continuously adjust the position and speed of micro stroke through a complex controller [2]. At present, its precision control at millimeter level is easy to realize, but it is difficult to control at micron level. In view of the above problems, an improved micro stroke hydraulic control system for the power slide of modular machine tool is proposed. In the system, the servo valve element is removed, and the servo motor and displacement amplifying hydraulic cylinder are added to improve the control of oil flow rate. In addition, the accumulator element is connected to the oil return chamber of the hydraulic cylinder to ensure that the pressure and volume of the oil in the process of micro stroke control are in a stable state. The feasibility of the micro stroke hydraulic control system of machine tool is verified by numerical simulation and experiment [3]. Hydraulic transmission control is a control method often used in industry. It uses hydraulic pressure to complete the process of energy transfer. Because of the flexibility and convenience of the hydraulic transmission control mode, the hydraulic control is widely valued in the industry. Hydraulic transmission is a subject that studies the pressure fluid as the energy medium to realize various mechanical and automatic controls. The hydraulic transmission uses this kind of component to form all kinds of control circuits needed, and then the transmission system which completes certain control functions is composed of several circuits to complete the transmission, conversion and control of energy.

\section{ANALYSIS OF HYDRAULIC CONTROL PRINCIPLE OF POWER SLIDE}

\section{A. Control requirement}

Modular machine tools are widely used in mass production. The main general-purpose component of the modular machine tool, the power slide, is used to realize the feed movement. It requires the hydraulic transmission system to complete the feed action: fast forward - the first working feed - the second working feed - stop - fast back - stop in situ. At the same time, it also requires the system to work stably and efficiently [4].

In order to meet the performance requirements of power sliding table, it is necessary to combine all hydraulic components organically to form a complete and effective hydraulic control circuit. In the power slide, the feed motion is actually driven by the hydraulic cylinder to complete the whole feed motion.

\section{B. Control principle}

The system uses the limited pressure variable displacement pump to supply oil, as shown in Fig.1. Use the travel valve to realize the change of fast forward and working forward. Two position two-way valve is used to realize the change of two working inlets, and the hydraulic control sequence valve is used to control the formation of fast forward and working oil inlet circuit. Check valve 5 separates the oil inlet circuit from the oil return circuit. Use relief valve as back pressure valve. In addition to protecting the variable displacement pump, check valve 2 is mainly used to make the control oil circuit have a certain pressure to control the start of the pilot solenoid valve. Use check valve 10 in order to connect the quick return circuit. Signal time relay with pressure relay.

Press the start button, the electromagnet ya1 is electrified, and the pilot valve core of the electro-hydraulic transfer direction valve moves to the right, thus causing the main valve core to move to the right, so that its left position is connected to the system. The main oil circuit is: oil inlet circuit: variable displacement pump 1 - check valve 2 - reversing valve 6 (left position) - travel valve 11 (lower position) - left chamber of hydraulic cylinder. Oil return circuit: right chamber of hydraulic cylinder - reversing valve 6 (left position) - check valve 5 - travel valve 11 (lower position) - left chamber of 
hydraulic cylinder, forming differential connection. When the slide quickly moves to the predetermined position, the travel stop on the sliding table presses down the valve core of the travel valve 11, so that the pressure oil enters the left chamber of the cylinder through the speed regulating valve 7 . At this time, the system pressure increases, check valve 5 closes, and the return oil returns to the tank through hydraulic control sequence valve 4 and back pressure valve 3 .

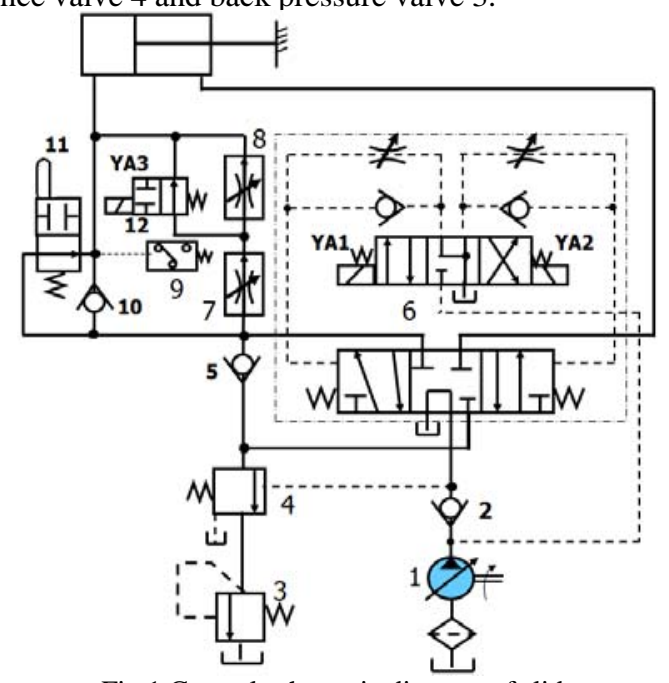

Fig.1 Control schematic diagram of slide

\section{Structure improvement}

The improved structure is composed of controller, servo motor, ball screw, displacement amplification hydraulic cylinder, platform base, actuating hydraulic cylinder, motion platform and accumulator [5]. The execution unit contains one or more execution cylinders. When the number of actuating cylinders is not one, the cylinders are connected in parallel, and the theoretical output force is the same. The controller can give different commands according to the positioning requirements of the moving platform: when the platform position command is up, the controller gives the servo driver and the motor forward rotation command to drive the ball screw and the displacement amplification hydraulic cylinder to move to the right, and part of the oil flows from the displacement amplification hydraulic cylinder to the lower chamber of the actuating hydraulic cylinder; at the same time, part of the oil in the upper chamber of the actuating hydraulic cylinder is squeezed into the accumulator, and the system There was a slight increase in pressure. On the contrary, when the platform position command is down, part of the oil flows back from the lower chamber of the actuating hydraulic cylinder to the displacement amplification hydraulic cylinder; part of the oil in the accumulator flows back to the upper chamber of the actuating hydraulic cylinder, and the system pressure drops slightly.

With the use of pressure limiting variable pump, the flow is automatically adjusted with the change of pressure, so that there is no power loss caused by overflow after the fast forward is turned into working, and the efficiency of the system is high. Using the stroke valve and the sequence valve to realize the fast forward and the working forward connection, not only simplifies the electrical circuit, but also acts reliably, and the conversion accuracy is higher than that of the electrical control type [6]. As for the connection between two working inlets, because of the low speed of both, the use of solenoid valve can fully ensure the connection accuracy.

\section{SIMULATION ANALYSIS OF HYDRAULIC SYSTEM}

\section{A. Model establishment}

AMESim is a multi-disciplinary complex system modeling and simulation platform. Users can build complex multidisciplinary system model on this single platform, and carry out simulation calculation and in-depth analysis on this basis, or study the steady-state and dynamic performance of any component or system on this platform. For example, in fuel injection, braking system, power transmission, hydraulic system, electromechanical system and cooling system. Engineering oriented positioning makes AMESim an ideal choice for R \& D in the automotive, hydraulic and aerospace industries. Engineering designers can design a system by using the integrated AMESim application library. All these models from different physical fields are strictly tested and verified by experiments. The LMS Imagine.Lab application specific solutions allow users focus on their design and engineering issues, without having to extensive time in creating and validating models. Suppliers can model, simulate and validate components early in the design process and provide a virtual functional mock-up of components or subsystem to the OEM. Manufacturers are then able to simulate the integration of suppliers' components and sub-systems in order to match the product functions specifications and validate key design choices [7].

The simulation model based on AMESim fully considers the nonlinear factors such as pipeline effect and gas state change, which can accurately reflect the open-loop performance of the system. The AMESim model of the system is shown in Fig.2. In order to verify the influence of each variable on the open-loop performance of the system, the orthogonal simulation tests are carried out on the variables such as the elastic modulus of the oil, the volume and pressure of the oil viscosity accumulator.

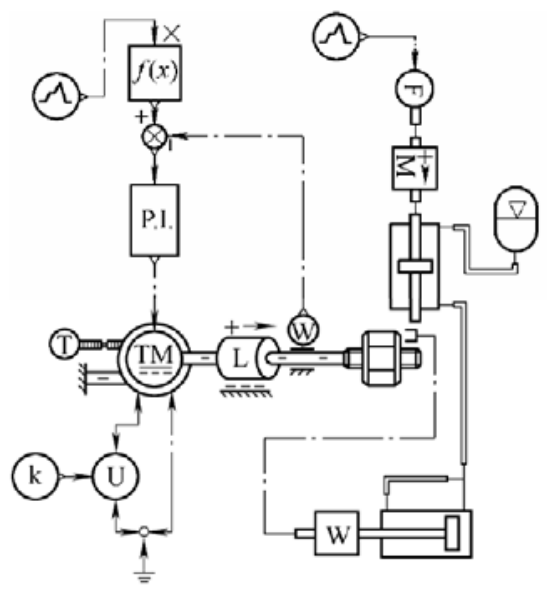

Fig.2 Hydraulic simulation model

\section{B. Result analysis}

The simulation results are shown in Fig.3-Fig.6. The elastic modulus and viscosity of the oil have obvious correlation with the system response: the larger the elastic modulus of the oil, the faster the system response; the smaller the elastic modulus of the oil, the slower the system response; the smaller the viscosity of the oil, the faster the system response; the larger the viscosity of the oil, the slower the system response. However, the accumulator pressure and volume have little effect on the open-loop response of the system. 


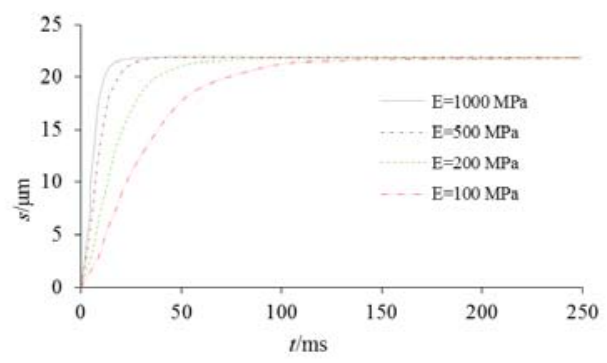

Fig.3 Open loop response of the system under different elastic modulus of oil

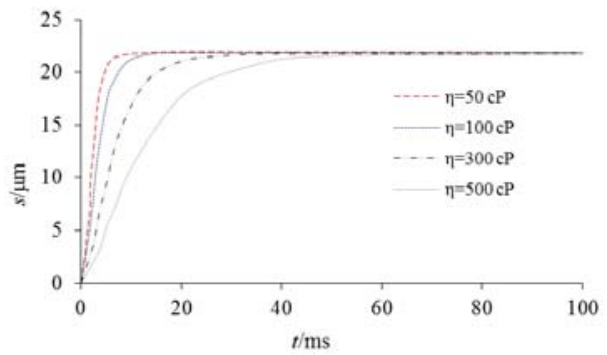

Fig.4 Open loop response of system under different oil viscosity

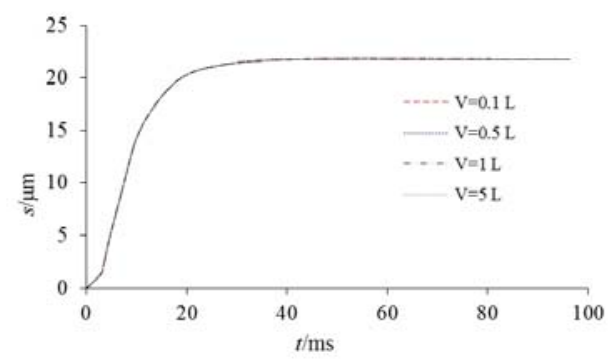

Fig.5 Open loop response of the system with different accumulator volumes

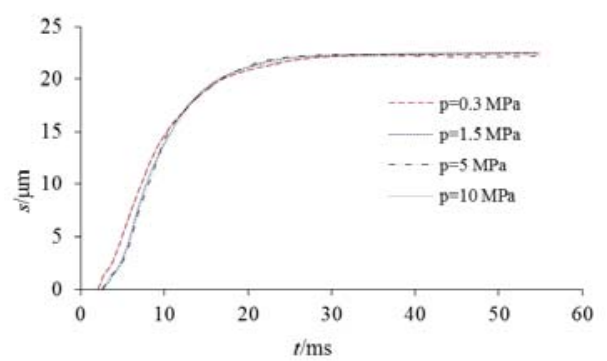

Fig.6 Open loop response of different accumulator capacity systems

The system identification method based on test data and MATLAB system identification toolbox has been widely used in practical engineering. The sweep signal is used as the input signal of the open-loop characteristic test to test the performance of the hydraulic system in each frequency range, which is more conducive to the parameter identification of the hydraulic system. Displacement amplification hydraulic cylinder is driven by servo motor, ball screw and other transmission components. Its motion curve is not a strict sweep curve, but does not affect its role in the system identification process. Sampling time $20-25 \mathrm{~s}$ data is used as system identification sample and 45-50s data is used as identification parameter verification sample.

\section{System performance verification}

In order to verify the precise positioning performance of the micro displacement control hydraulic system, the control experiment was carried out on the micro displacement integrated control test-bed, and the parameters of the controller were adjusted according to the identification model. The comparison results of experiment and simulation are as shown in Fig.7, which shows that it has good matching performance. For the micro displacement control system, the hydraulic part of the system is a second-order system, while the part from the controller to the displacement amplifier hydraulic cylinder can generally be regarded as a first-order integral link. For the low order system, PD controller can be used as the partition position controller, and the parameter setting process of PD controller is as follows.

(1) Square wave signal is used as system input to test the open-loop dynamic performance of the system.

(2) The closed-loop step signal position control parameters of the system are debugged, and the proportional system is set to 0 . Set the initial value of $\mathrm{Kd}$ to a smaller value, gradually increase the value of $\mathrm{Kd}$ until the system has an equal amplitude oscillation, record the Kd value at this time, and then set the value of $\mathrm{Kd}$ to 0.7 times of the value.

(3) Gradually increase the differential coefficient Kd until the damping oscillation occurs in the system, and then gradually reduce the value of $\mathrm{Kd}$ to eliminate the oscillation.

Limited pressure variable displacement pump, with the change of load and output different flow of oil, to adapt to the requirements of fast movement and working feed (slow speed). The check valve, in addition to preventing the oil backflow of the system and protecting the variable displacement pump, mainly makes the control oil circuit have a certain pressure to control the start of the pilot valve.

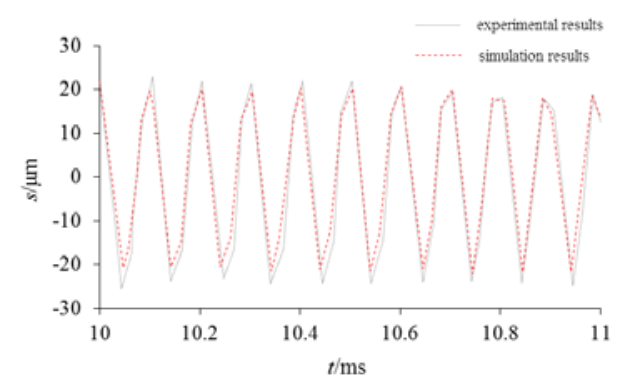

Fig.7 The comparison results of experiment and simulation

\section{CONCLUSION}

In recent years, with the application of computer technology, information technology, automatic control technology and new materials, hydraulic transmission has made new development, which makes the hydraulic system and components develop in the direction of high pressure, high speed, high precision and high efficiency, and has made new achievements in improving proportional control, servo control, digital control and other technologies. The development direction of hydraulic system is to create new energy-saving, micro components, high combination, integration, modularization and microelectronics, and move towards intelligence. Hydro pneumatic technology is one of the fastest 
developing technologies in mechanical equipment. Especially in recent years, with the combination of microelectronics and computer technology, the hydraulic pneumatic technology has entered a new stage of development. At present, it has been widely used in various fields of industry. In recent years, with the development of microelectronics and computer technology and the further improvement of manufacturing technology of hydraulic and pneumatic components, the hydraulic and pneumatic technology not only plays an important role as a basic transmission form, but also becomes an important control means with excellent static and dynamic performance.

\section{ACKNOWLEDGEMENT}

The paper is supported by Scientific Research Foundation Project of Binzhou University (No. BZXYG1612).

\section{REFERENCES}

[1] F. Xijing. "AMESim simulation technology and its application in hydraulic system," Hydraulic Pneumatic and Sealing, vol.32, pp.206-209, October 2015.
[2] L. Liu-min, H. Guiyun. "Research status and development trend of hydraulic system fault diagnosis technology," Computer Simulation, vol.33, pp. 234-237, August 2016.

[3] L. Hongsheng. "Development and research status of hydraulic free piston engine," Journal of Mechanical Engineering, vol. 31, pp. 407-411, January 2014.

[4] L. Qiaohua. "Hydraulic energy system of large airliner," Journal of Automotive Engineering, vol. 01, pp. 464-468, April 2014.

[5] Q. Canwen. "theoretical analysis of flow pulsation of axial piston pump," Lubrication and Sealing, vol.3, pp. 45-47, June 2018.

[6] Z. Zhiguo, P. Yugang. "Inverted pendulum control system based on PID neural network," Mechanical Design and Manufacturing, vol.24, pp.208-215, July 2015.

[7] W. Kesheng, Z. Leiqiang. "Dynamic simulation of hydraulic system based on Simulink," Computer Simulation, vol.28, pp.342-347, April 2013. 